African Crop Science Journal by African Crop Science Society is licensed under a Creative Commons Attribution 3.0 Uganda License. Based on a work at www.ajol.info/ and www.bioline.org.br/cs DOI: http://dx.doi.org/10.4314/acsj.v26i3.1

\title{
GENOME WIDE ANALYSIS OF NAC TRANSCRIPTION FACTORS AND THEIR EXPRESSION PATTERN DURING HIGH TEMPERATURE AND DROUGHT STRESS IN GROUNDNUT
}

\author{
B. SUCHITHRA ${ }^{1,2}$, V.R. DEVARAJ ${ }^{2}$ and R. NAGESH BABU ${ }^{1}$
}

${ }^{1}$ Post Graduate Department of Biochemistry, Maharani's Science College for Women, Bangalore 560001, India ${ }^{2}$ Department of Biochemistry, Central College, Bangalore University, Bangalore 560001, India

Corresponding author: nageshbabur@gmail.com

(Received 4 December, 2017; accepted 26 July, 2018)

\begin{abstract}
NAC (NAM, ATAF1/2 and CUC2) is a prime plant specific transcription factor, which plays a pivotal role in stress signaling. Excavating a relatively large number of NAC TFs under complex environmental cues and understanding their molecular basis, remains a challenge. The objective of this study was to analyse a total of 76 NAC transcription factors of which 38 were from Arachis duranensis (AdNAC) and Arachis ipaensis (AiNAC) for phylogeny, chromosomal location, conserved motif identification including membrane bound NTLs (NAC trans-membrane like), promoter analysis and expression profiles under high temperature and drought stress. The study led to the identification of eight membrane bound NTLs, such as AdNAC26, AdNAC36, AiNAC16, AiNAC17, AiNAC37, AdNAC14, AiNAC12, and AiNAC29, and revealed that majority of NAC proteins had four NAC domain- containing conserved motifs and were localised at the nucleus. The study also reveals $A d N A C 21$ and AiNAC3 as positive regulators under both stress conditions. Our results provide a basis for selection of promising stress- responsive NAC candidates for further functional analysis, leading to development of transgenics with improved productivity of groundnut varieties under drought and high temperature.
\end{abstract}

Key Words: Conserved motifs, NTL, phylogenetic tree, RT- qPCR

\section{RÉSUMÉ}

NAC (NAM, ATAF1/2 et CUC2) est un facteur spécifique primordial dans la transcription chez la plante, qui joue un rôle principal dans la signalisation des stresses. Fouiller un nombre relativement important de NAC TFs sous le complexe des signaux environnementaux et comprendre leur base moléculaire, demeurent un défi. L'objectif de cette étude était d'analyser un total de 76 facteurs de transcription desquels 38 sont de Arachis duranensis $(A d N A C)$ et Arachis ipaensis (AiNAC) pour la phylogénie, la localisation chromosomique, l'identification du motif conservé y comprises la membrane liée NTLs (semblable à NAC transe-membrane), analyse du promoteur et les profils d'expression sous le stress de haute température et de sécheresse. L'étude a conduit à l'identification de huit membranes NTLs liées, telles que AdNAC26, AdNAC36, AiNAC16, AiNAC17, AiNAC37, AdNAC14, AiNAC12, et AiNAC29, et a révélé que la majorité des protéines NAC ont quatre domaines NAC- contenant des motifs conservés et sont localisés dans le noyau. L'étude a aussi révélé AdNAC21 et AiNAC3 comme régulateurs positifs sous les deux conditions à la fois. Nos résultats ont fourni une base pour la sélection des NAC candidats donnant de réponses satisfaisantes aux stresses pour une analyse fonctionnelle avancée, conduisant au développement des transgéniques avec des variétés d'arachide à rendement amélioré sous la sécheresse et une haute température.

Mots Clés: Motifs conservés, NTL, arbre phylogénétique, RT- qPCR 


\section{INTRODUCTION}

Modern agriculture faces several challenges that include global climate change, complex field environment, and a combination of abiotic stresses which limit crop production and food security. It is now well established that abiotic stress is regulated by transcription factors (TFs). Certain TFs enable the plant to withstand unfavourable conditions, thereby becoming potential candidates for crop breeding. Such TFs represent key molecular switches orchestrating the regulation of plant developmental processes. NAC proteins constitute one of the largest transcription factor (TF) families, and are characterised by a well-conserved $\mathrm{N}$ terminal NAC domain (Puranik et al., 2012). The NAC domain, comprising nearly 160 amino acid residues, can be divided into five sub domains (A to E) based on its motif distribution (Ooka et al., 2003). The highly conserved sub-domains $C$ and D may be responsible for binding to DNA, sub-domain A may be involved in homo and hetero-dimerisation, and the divergent subdomains $\mathrm{B}$ and $\mathrm{E}$ may be implicated in the functional diversity of NAC proteins (Ooka $e t$ al., 2003; Chen et al., 2011).

NAC coding genes are themselves regulated by other factors such as ABREs, DREs, microRNAs or alternative splicing, and by the process of post translational modification (Puranik et al., 2012). As an additional feature, some NAC proteins comprise of a helical transmembrane motif for anchoring to plasma membrane or endoplasmic reticulum. NAC proteins have been demonstrated to play a role in a wide range of plant developmental processes, including lateral root formation (He et al., 2005), shoot branching (Chuanzao et al., 2007), flowering (Sablowski et al., 1998) and leaf senescence (Guo and Gan, 2006).

Ren et al. (2017) demonstrated the function of SiNAC1 as a positive regulator in leaf senescence. Numerous NAC domain proteins also play important roles in various biotic and abiotic stress responses such as drought (Jeong et al., 2010), salinity (Zheng et al., 2009), cold weather (Aslam et al., 2012), fungal and bacterial pathogens (Wang et al., 2009). Availability of complete plant genome sequence; followed by extensive investigation resulted in identification of more than 100 NAC genes in Arabidopsis, rice, soybean, foxtail millet, Chinese cabbage, 74 in grape and 88 in pigeonpea (Zhenying et al., 2015). Although the genes encoding transcription factors account for a little portion in the whole genome, transcription factors are important in the regulated networks (Hobert et al., 2008). Thus, understanding the complex mechanism of drought and high temperature tolerance is important for agriculture production.

Groundnut (Arachis hypogaea L.) is an oilseed crop cultivated worldwide and is one of the major grain legumes in tropical and subtropical regions. To date, 80 species in genus Arachis exist and have been classified into nine taxonomic sections (Bertiol et al., 2011). Wild species are diploid, while cultivated groundnut is allotetraploid (AABB). Based on morphology, cytology, fertility of the interspecific hybrid and molecular studies, the wild ancestral species of cultivated groundnut are generally considered to be duranensis and ipaensis, which contribute the A and B subgenomes (Kochert et al., 1996; Seijo et al., 2007). The objective of this study was to analysegene structure, promoter analysis, evolutionary significance and expression profiles of NAC TFs using the available assembled genome. The results thus provide a comprehensive genome-wide analysis of NAC proteins and a preliminary investigation of specific NAC proteins potentially involved in drought and high temperature response. Through this investigation, we aim to provide insight into the characterisation, thereby decoding the function of NAC genes in groundnut. 


\section{MATERIALS AND METHODS}

Sequence retrieval, phylogenetic analysis and sub-cellular localisation. The NAC domain containing gene and protein sequences were retrieved from the Plant Transcription Factor Database ver. 2.0 and Arachis genome (Peanut Base). The sequences were analysed for the HMM (Hidden Markov Model) profile of the NAC domain, downloaded from the Pfam database using HAMMER (ver. 3.0). All redundant sequences were filtered and curated for the presence of conserved NAC domain, with the help of Pfam (http://pfam.sanger. ac.uk/), SMART (http://smart.embl-heidelberg. de/) and InterProScan (http://www.ebi.ac.uk/ Tools/InterProScan/). The length, molecular weight and $\mathrm{pI}$ of each deduced polypeptide were predicted using ExpasyProtParam (http:/ /web.expasy.org/protparam/). Furthermore, WOLF PSORT (http://www.genscript.com/ psort/wolf_psort.html) tool was used to predict the sub-cellular localisations. NAC protein sequences were imported to BioEdit (Hall, 1999) and were aligned by ClustalW (v7.2.5). MEGA v6.06 was used to construct the unrooted-phylogenetic tree, using neighbor-joining $(\mathrm{N}-\mathrm{J})$ method (bootstrap, 1000 replicates) based on the pairwise gap deletion method (Tamura et al., 2013). Another phylogenetic tree was built for the illustration of the relation between NACs from groundnut (Arabidopsis and Glycine max).

Genomic structure, chromosome location and identification of conserved motifs. A Gene Structure Display Server from the Center for Bioinformatics, Peking University, was used to display the intron exon junctions (http:/ /gsds.cbi.pku.edu.cn/index.php). The genomic and mRNA sequences of these NACs were downloaded and used as query for generating its gene structure. A number of introns and exons were estimated based on this alignment and confirmed by the coordinated sequences. The chromosomal location of $A d N A C$ and $A i N A C$ genes were obtained from Peanut base website (http://peanutbase.org/) and the map was generated using MapInspect (http:// mapinspect.software.informer.com/). The MEME Suite tool v4.9.1 (http://meme.nbcr. net/meme) (Bailey et al., 2009) and STRING 10 (http://string.embl.de/) were used for conserved motifs analysis and gene ontology studies, respectively. Furthermore, TMHMM Server ver.2.0 (http://www.cbs.dtu.dk/ services/TMHMM/) was used to predict the membrane bound NAC members. 1kilo bases of upstream DNA sequence of the initiation codon (ATG) of genes was retrieved from Peanut Base and the cis-acting regulatory DNA elements (cis-elements) in the promoter regions of NAC genes were identified using PlantCARE tool (http://bioinformatics. psb.ugent.be/webtools/plantcare/html/).

Plant materials and abiotic stress treatment. Seeds of groundnut (ICGV1999) were surface sterilised and sown in mixture containing 3:1 ratio of autoclaved cocopeat and acid washed sand in the plastic germinating trays (PVC, Size: $54 \mathrm{~cm} \times 27.99 \mathrm{~cm}$ ). The trays were sprayed with modified Hoagland media daily and maintained under controlled conditions $\left(28{ }^{\circ} \mathrm{C}\right.$ day per $25^{\circ} \mathrm{C}$ night with a $12 \mathrm{hr}$ light per $12 \mathrm{hr}$ dark photo period in the poly house). After germination, 10 days old seedlings were exposed to drought stress by withholding water for 5 days and for high temperature stress, seedlings were exposed to $42{ }^{\circ} \mathrm{C}$ for $2 \mathrm{hr}$ (induction), followed by $48^{\circ} \mathrm{C}$ for $6 \mathrm{hr}$. Control and stress exposed tissues were harvested immediately and stored at -80 ${ }^{\circ} \mathrm{C}$ for further analysis.

Expression analysis of NAC genes. Total RNA was isolated using TRizol (Invitrogen) and treated with RNAase-free DNAase I (Promega) from the control and stress tissues (shoot, cotyledon, leaf, stem and root), according to the manufacturer's (Thermo Fisher Scientific) instructions. The RNA concentration was measured by Nanodrop 2000 (Thermo Scientific). cDNA was 
synthesized by reverse transcriptase with 500ng of total RNA using PrimeScript RT Reagent Kit (Takara) according to the manufacturer's instructions. Gene specific primers were designed using Primer3 software (Table 1). qRT-PCR reactions were performed using SYBR Green PCR Master mix (Takara) on Lightcycler96 Real time PCR (Roche), which include $2 \mathrm{ml}$ cDNA, 1x SYBR Green Master mix, $0.5 \mathrm{ml}$ gene specific forward primer $(10 \mathrm{mM}), 0.5 \mathrm{ml}$ reverse primer $(10$ $\mathrm{mM}$ ), and $7 \mathrm{ml}$ sterile water. The NAC expression was normalised against actin as reference gene. The qPCR reactions conditions were as follows: $95^{\circ} \mathrm{C}$ for $10 \mathrm{~min}$, followed by 40 cycles of $95{ }^{\circ} \mathrm{C}$ for $15 \mathrm{~s}, 55^{\circ} \mathrm{C}$ for $30 \mathrm{~s}$ and $72{ }^{\circ} \mathrm{C}$ for $15 \mathrm{~s}$. All reactions were run in three technical and two biological replicates. The relative difference in expression for each experiment was analysed using $2^{-\Delta \Delta C T}$ method. The data were imported; and using Morpheus software (software.broadinstitute.org/ morpheus/), heat map was generated. The average of two biological replicates was used to get each expression value.

\section{RESULTS AND DISCUSSION}

In-silico analysis of NAC TFs. To identify the NAC TFs, we retrieved all the predicted NAC gene and protein sequences from Plant TFDB and Peanut Base (http://peanutbase.org). The keyword, HMM profile and BLAST search predicted from each genome encodes about 38 NAC proteins and 76 NAC genes were identified from both A. duranensis and A. ipaensis. They were named from AdNAC1 to AdNAC 38 , and AiNAC1 to AiNAC38, respectively. The average polypeptide length was found to be 347.1 aa residues, with AdNAC17 (158 aa) being the shortest and AiNAC37 (698 aa) being the longest. The pI values ranged from 4.43 to 9.46 . The subcellular localisation results revealed that only 9 out of 76 NACs were localised to cytoplasm; and the rest situated in the nucleus. The multiple alignment of AdNACs, AiNACs and NACs from Arabidopsis and Glycine max indicated that all the NACs shared a highly conserved N-terminal DNA binding NAC domain, consisting of five consensus subdomains (A-E), and a variable C- terminal transcriptional regulation domain. Additionally, a conserved bipartite nuclear localisation signal was also found in the D sub-domain of groundnut NACs, suggesting that these NACs are localised to the nucleus (Fig. 1).

To examine the structure and phylogenetic relationships of NAC TFs identified in our study, a combined phylogenetic tree was constructed with the aligned NAC domains from groundnut, Arabidopsis and Glycine max). The phylogenetic tree showed that the NAC TFs can be classified into nine major groups: Group I (13 NACs), Group II (7), Group III (17), Group IV (6), Group V (15), Group VI (4), Group VII (2), Group VIII (4) and Group IX (10). Phylogenetic tree constructed from the AdNACs, AiNACs and AdNAC- AiNACs together are depicted in Figures 2 and 3, respectively. The relationship among the $76 \mathrm{NAC}$ genes investigated through constructing phylogenetic trees using Neighbour Joining method and it revealed that several pairs of NAC proteins had a high degree of homology in the terminal nodes of each

TABLE 1. List of primers for qRT-PCR

\begin{tabular}{lllc}
\hline Gene name & Forward (5'-3') & Reverse (5'-3') & $\begin{array}{l}\text { Product } \\
\text { Size }\end{array}$ \\
\hline AdNAC21 & TCCACCAGGTTTCAGATTCC & AAGCCTTCTTTGGCAAATCC & 149 \\
AiNAC3 & TGGAGTGAAGAAAGCCCTTG & GCATAGCACCCACTCATCAA & 151 \\
\hline
\end{tabular}




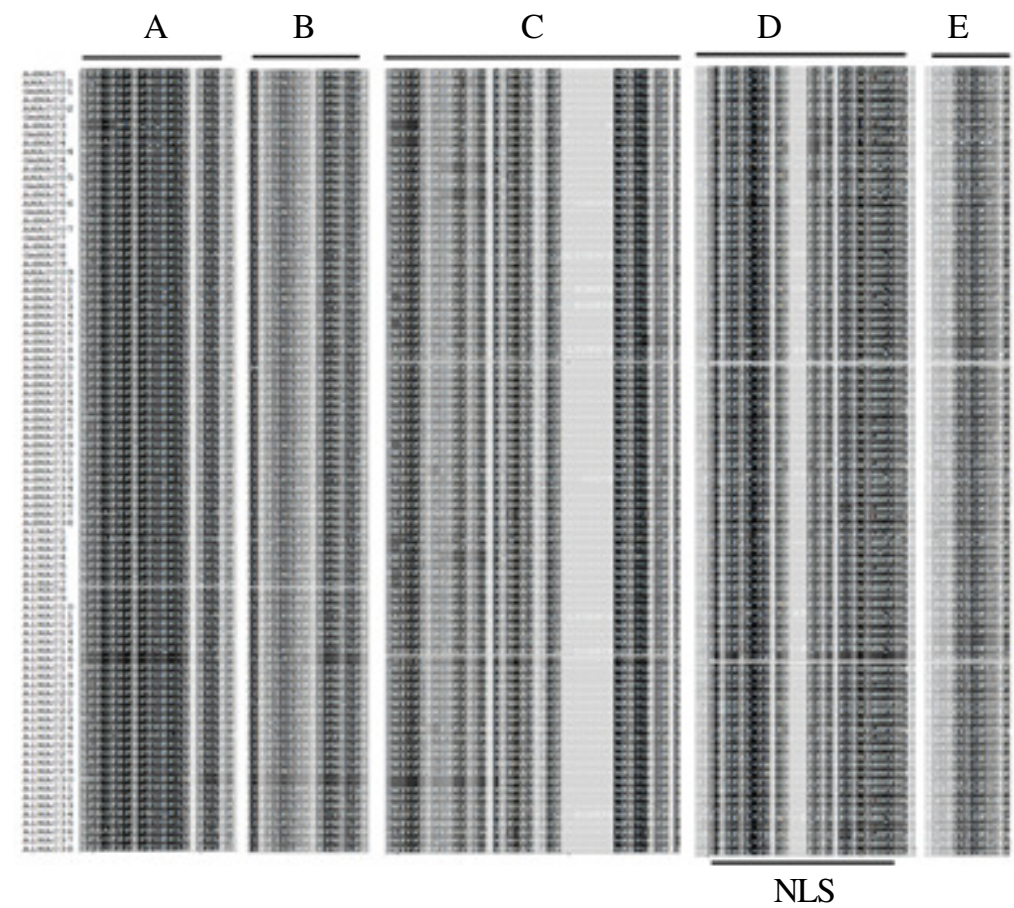

Figure 1. Multiple alignment of 76 NACs of groundnut, Arabidopsis and Glycine max. Conserved NAC domain and sub-domains (A-E) are indicated by thick blue line and black thin lines, respectively, above the sequences. The putative nuclear localisation signal (NLS) is shown by a black line below the sequence.

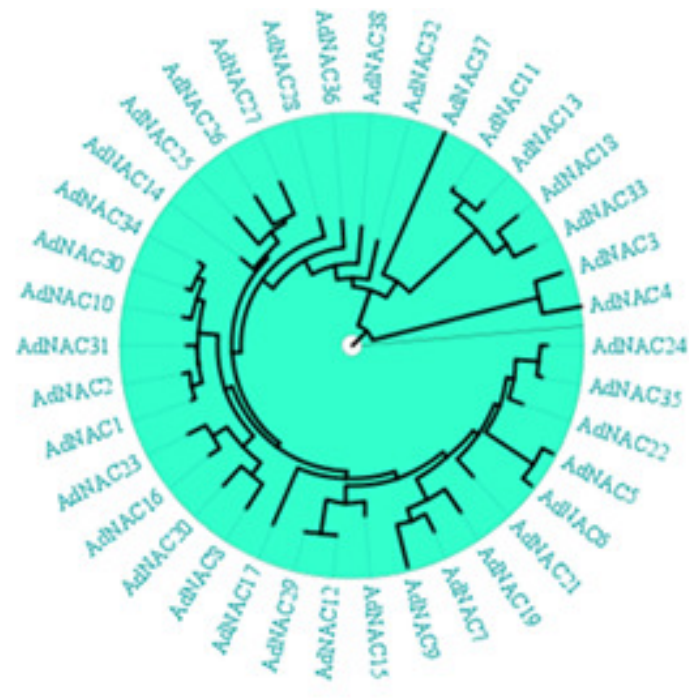

$\overline{0.3}$

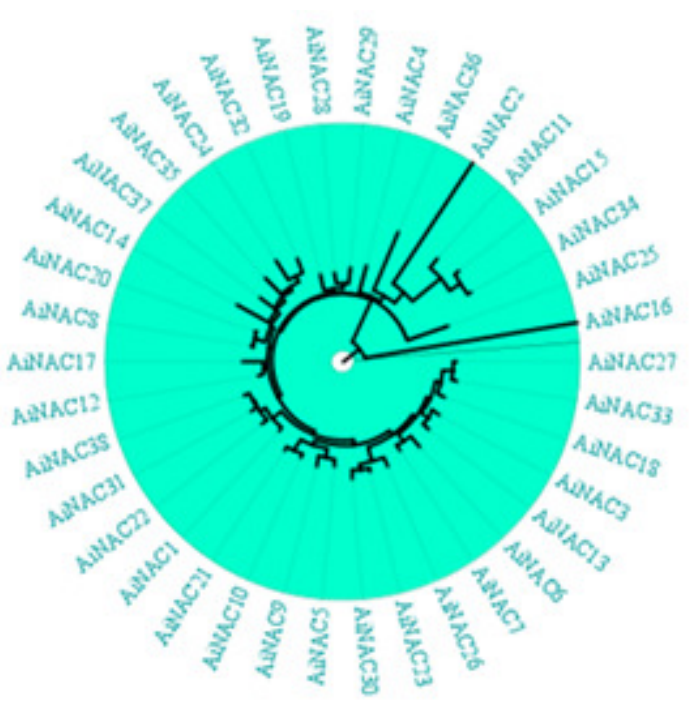

$\overline{0.5}$

Figure 2. Unrooted phylogenetic tree constructed using the neighbor-joining (NJ) method, and the bootstrap test was carried out with 1,000 iterations representing the relationships between AdNACs and AiNACs. 


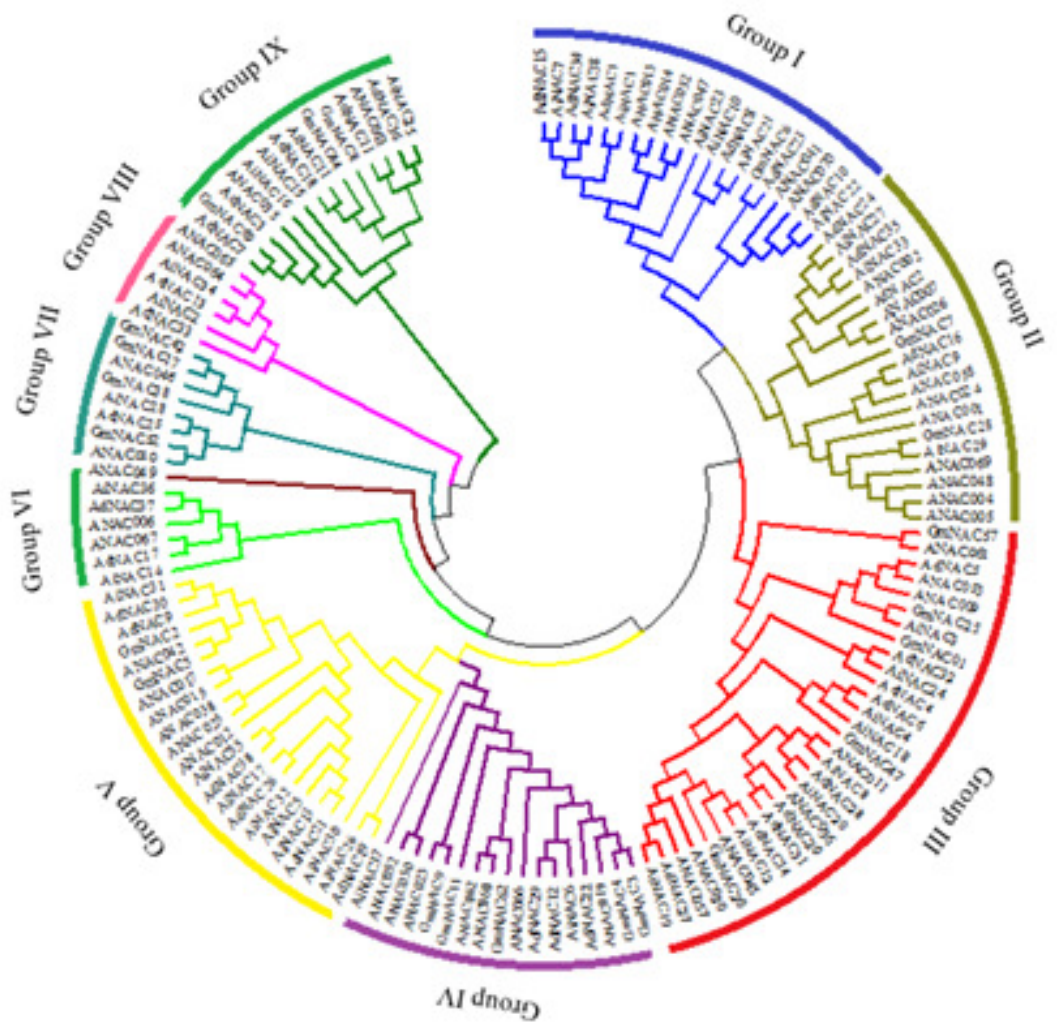

Figure 3. Unrooted phylogenetic tree constructed using the neighbor-joining (NJ) method, and the bootstrap test carried out with 1,000 iterations representing the relationships between the Peanut, Arabidopsis and Glycine max NAC domain proteins.

subfamily, suggesting they share similar functions but diverged by gene duplication.

Chromosomal distribution and gene structure of NAC genes. AdNAC and AiNAC genes were mapped to the groundnut genome according to their position information from Peanut Base. The genome of groundnut comprises of 20 chromosomes (10 from duranensis and 10 from ipaensis) varying in length; chromosome 8 (48.94 $\mathrm{Mb})$ being the shortest, and chromosome 3 (133.14 Mb) being the longest in A. duranensis. For $A$. ipaensis, the shortest was chromosome 2 $(108.64 \mathrm{Mb})$ and the longest was chromosome 5 (149.44 Mb) (Fig. 4). In-silico mapping depicted an uneven distribution of the genes on all the chromosomes. Among all, chromosome 3 contained the highest number of NACs (26\%); while only one gene was located on chromosome $4(0.026 \%)$. There was no positive correlation between chromosome length and number of NAC genes. The ends of chromosome exhibited stronger synteny than the central regions.

The gene structures were investigated through genomic annotation, to determine the structural diversity. All NAC genes harbored at least two exons, except AiNAC16 being the shortest not having intron. In addition, a separate phylogenetic tree was generated from the complete protein sequences of all the NAC genes (Fig. 5). It is well known that gene structural diversity is a possible mechanism for the evolution of multi-gene families (Nagy et al., 2012). Investigation of gene structures 


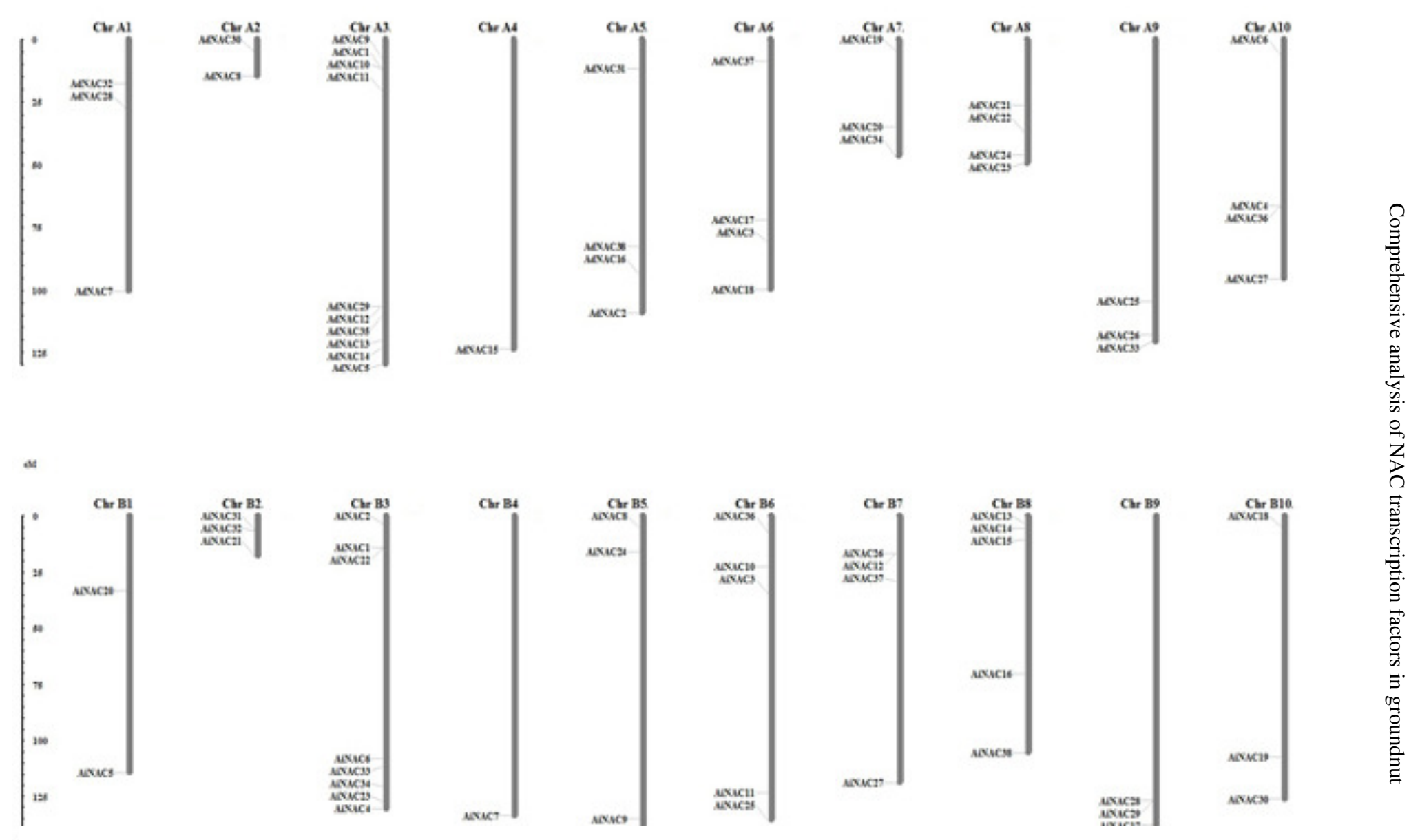

Figure 4. Distribution of 76 NAC genes on Peanut chromosomes, and physical locations of each NAC gene on the ten chromosomes from each species (positions in $\mathrm{cM}$ ). 


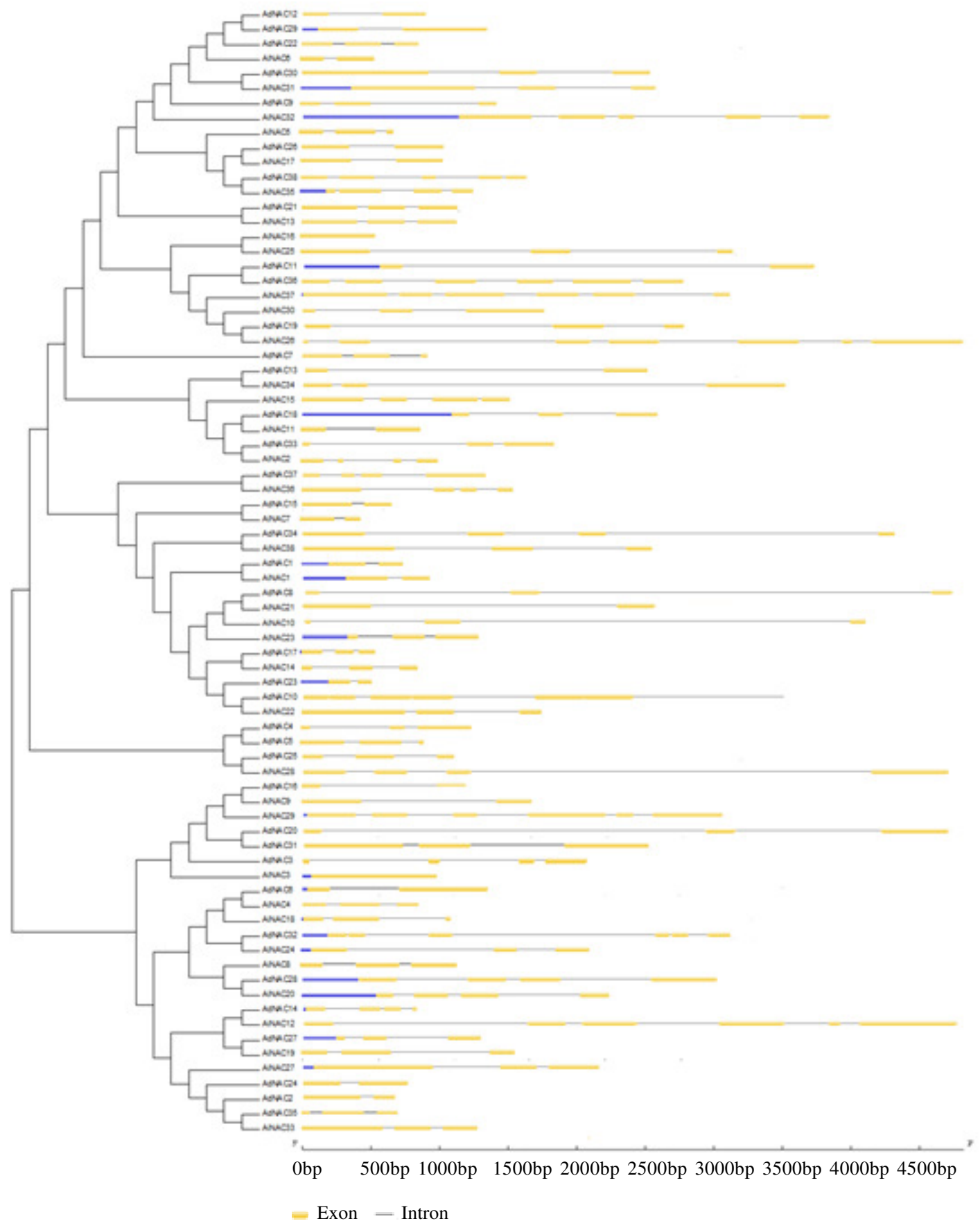

Figure 5. Phylogenetic relationship and gene structure of the $N A C$ genes. The phylogenetic tree was constructed with MEGA6.0 using the neighbor-joining (NJ) method with 1,000 bootstrap replicates based on a multiple alignment of 76 amino acid sequences of NAC genes from Arachisduranensis\&Arachisipaensis. Exon/ intron structure of $N A C$ genes are represented by boxes and black lines, respectively. 
revealed that most closely related members in the same subfamilies shared similar exon/ intron structure in terms of intron number and exon length suggesting these members may be evolved early and represent the ancestral form.

Identification of conserved motifs, membrane bound NTLs. The MEME (Multiple Expectation Maximisation for Motif Elicitation) server was used for exploring motif distribution in 38 AdNAC and 38 AiNAC proteins. Nine different conserved motifs were identified, of which most of them had at least four NAC domain-encoding motifs, and 54 shared a highly conserved typical NAC domain containing five consensus sub-domains (motifs 2, 4, 1, 5 and 3) in the same order (Fig. 6). The motif sequence logos are depicted in Figure 7. All well-known NAC domain proteins bind specifically to the CATGTG motif of the promoter region (Tran et al., 2004) or act as a functional motif or activation domain (Oh et al., 2005). Multiple sequence alignment and identification of conserved motifs indicated that most of the NAC proteins possessed A to E sub-domains in the $\mathrm{N}$ termini that conferred DNA-binding activities. The motif composition of NACs may provide clues for further functional analysis of this TF.

Functional conservation within a sub-family serves as an initial platform in facilitating a better understanding of the structure-function relationship between individual members. However, the biological significance of most of the putative motifs remains to be elucidated. Prediction of functional protein association network of NAC in Arabidopsis using STRING programme revealed the interaction of NAC083 (AdNAC15) with VND1, VND7, NAC1, NAC41, ANAC026 and NAC007; XND1 (AdNAC5) with NAC073; NAC010; NAC090 (AiNAC14) with NAC044; NAC036; BTF3 (AiNAC2) with NACA2; and AT3G12390 (Fig. 8).
Gene ontology (GO) annotation of NAC showed the involvement in different biological processes, cellular component and molecular functions (Fig. 9). Gene ontology annotation reveals that most of these proteins were predicted to be involved in response to stress as well as cellular, metabolic and biosynthetic processes; in addition to transcription regulatory activity. Furthermore, cellular component analysis revealed the localisation of these gene products in nucleus.

The membrane transcription factors are stored in their dormant forms in association with the intracellular membranes (Kim et al., 2010). During abrupt environmental changes, they are released from the membranes through proteolytic cleavage events and enter the nucleus, and regulate the expression of genes involved in perception of stress signals (Kim et al., 2010). Among 76 NACs (38 AdNACs and 38 AiNACs), 08 members (AdNAC14, 26, 36, AiNAC16, 17, 29 and 37) were identified as membrane-associated NTLs (Table 2), five (AdNAC26, AdNAC36, AiNAC16, AINAC17 and AiNAC37) and three (AdNAC14, AiNAC12 and AiNAC29) members contain one and two TMHs, respectively.

The phylogenetic tree constructed with membrane associated NTLs identified from groundnut, Arabidopsis and rice, indicated that the groundnut NTLs were scattered into different groups (Fig. 10). Several putative trans-membrane helices have been identified in other plant species, such as chickpea (Ha et al., 2014), Glycine max (Le et al., 2011), Arabidopsis (Kim et al., 2010), rice (Kim et al., 2007), maize (Shiriga et al., 2014), potato (Singh et al., 2013), foxtail millet (Puranik et al., 2013), chinese cabbage (Liu et al., 2014) and tomato (Kou et al., 2014). Two out of 11 putative GmNTLs from soybean, and 4 out of 8 CaNTLs from chickpea possess two TMHs; whereas all the NTLs predicted in other plant species possess only one TMH. This suggested that the existence of doubled TMHs might be specific to leguminous plants. A 


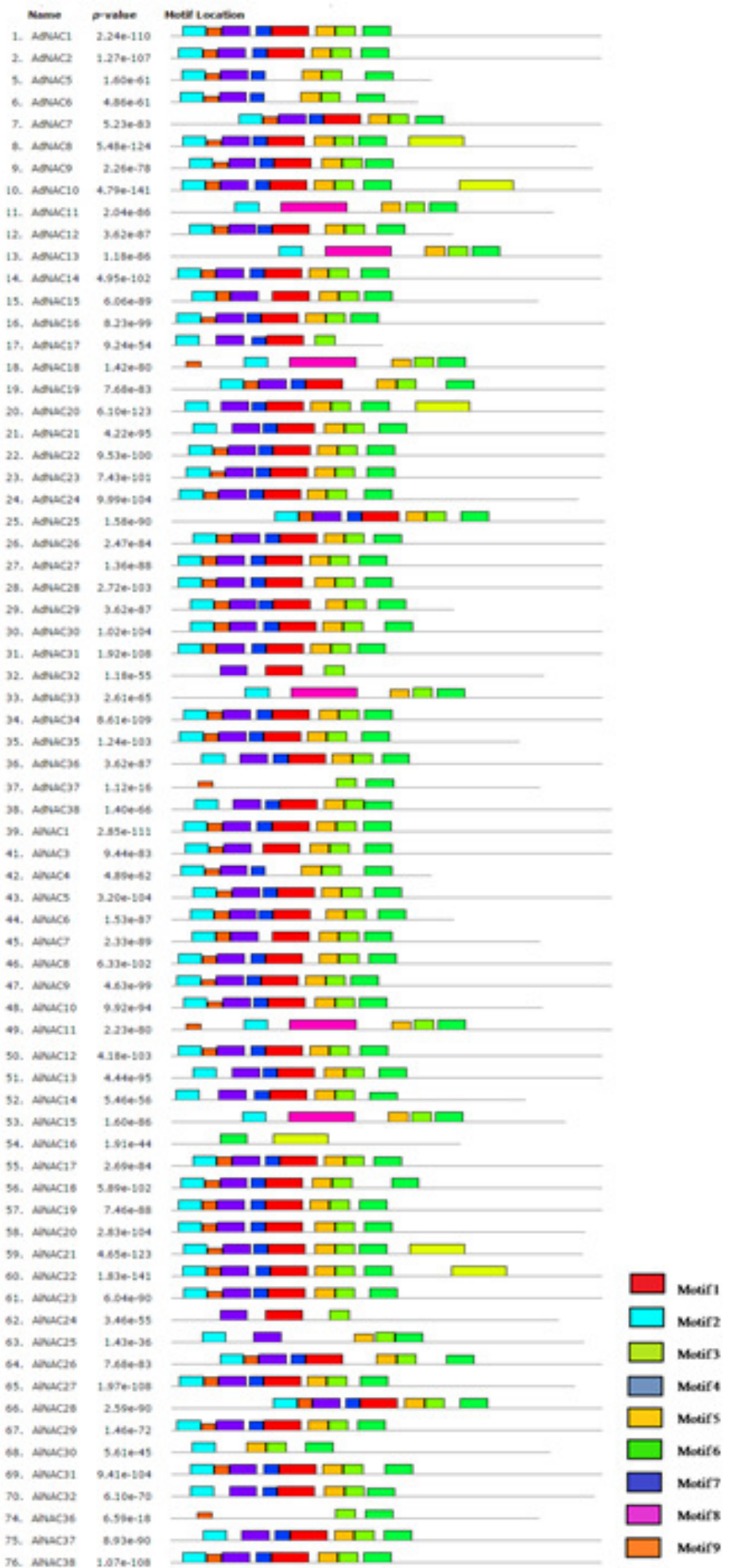

Figure 6. Schematic representation of conserved motifs in the AdNAC and AiNAC proteins predicted by MEME. Each motif is represented by a number in the coloured box. The black lines represent non- conserved sequences. 


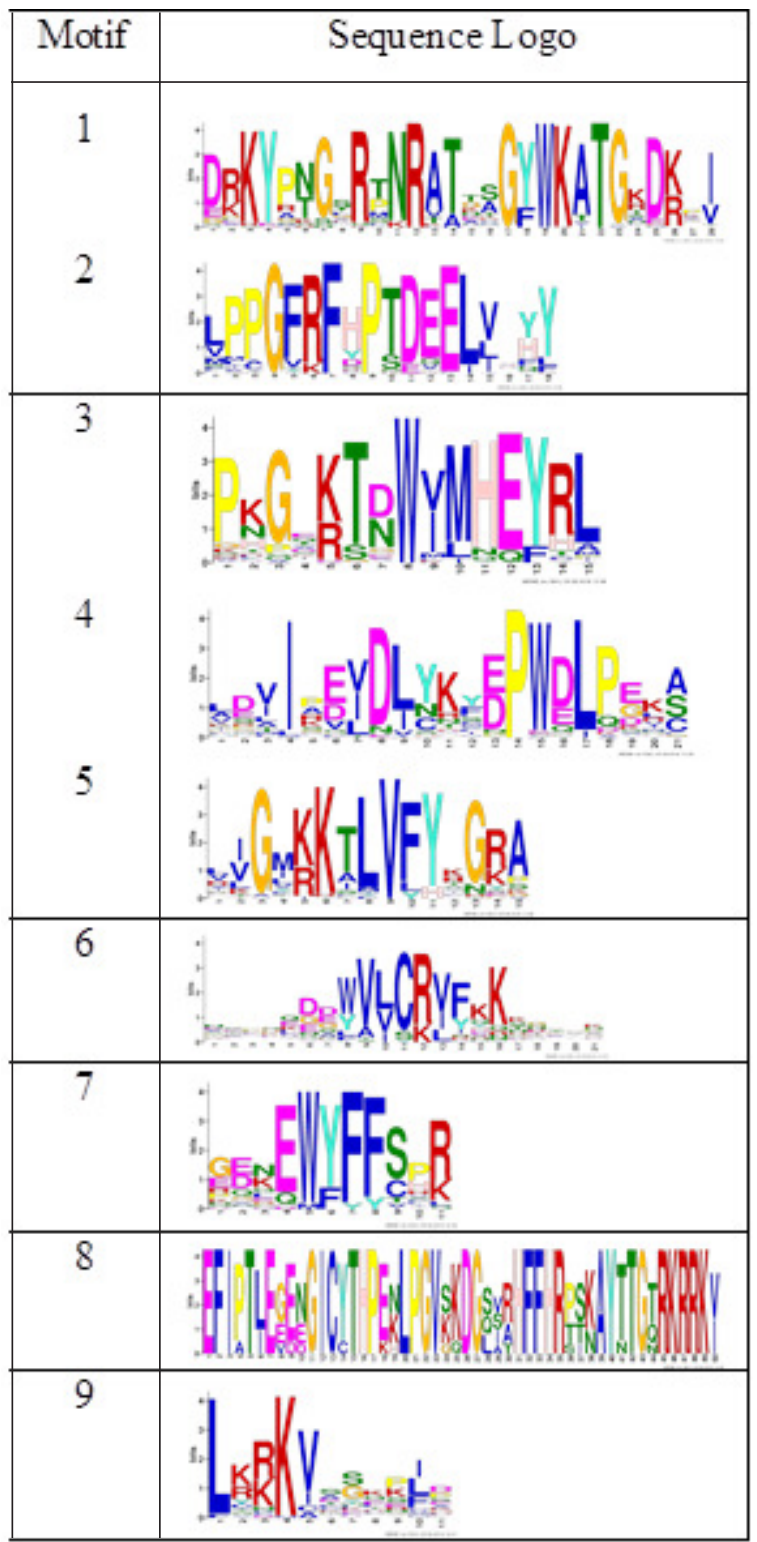

Figure 7. Conserved motif logos identified using MEME tool.

phylogenetic tree constructed from the NTLs from groundnut, Arabidopsis (NTLs/ ANACs) and rice (OsNTLs/ ONACs) indicated that the groundnut NTLs were scattered into four major groups. Membrane bound NAC proteins have been implicated as major players in biotic and abiotic stress response affecting major physiological processes like flowering, seed germination, leaf senescence and cell division (Mohammed et al., 2013). In Arabidopsis, a membrane bound NAC and NTL6, was activated upon cold stress, as the membrane fluidity changed and induced the expression of pathogenesis related proteins. In addition, plant hormone ABA activates the NTL6 (Seo et al., 2010), thus indicating the involvement 


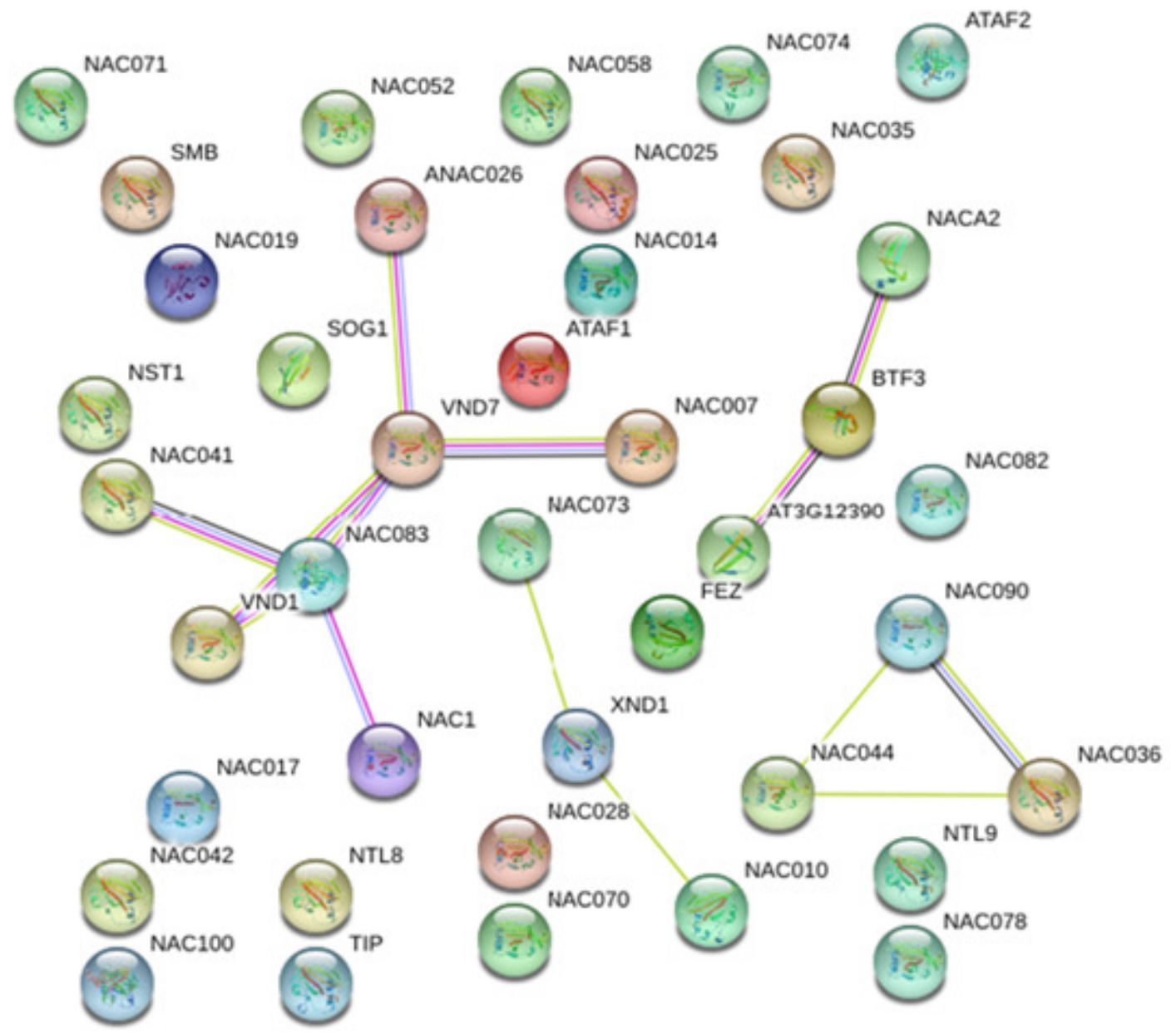

Figure 8. Functional protein association network of groundnut NAC proteins in Arabidopsis.

TABLE 2. Putative membrane- bound groundnut NTLs

\begin{tabular}{|c|c|c|c|c|c|}
\hline Gene name & $\begin{array}{c}\text { Membrane } \\
\text { bound member }\end{array}$ & Length (aa) & $\begin{array}{l}\text { Transmembrane } \\
\text { sequences }\end{array}$ & $\begin{array}{l}\text { Expected number } \\
\text { of AAs in TMHs }\end{array}$ & $\begin{array}{l}\text { Expected number } \\
\text { first } 60 \mathrm{AAs}\end{array}$ \\
\hline AdNAC14 & $\begin{array}{l}\text { AdNTL1, } \\
\text { AdNTL2 }\end{array}$ & 633 & $\begin{array}{l}525 \ldots . .544 \\
612 \ldots .631\end{array}$ & 40.88286 & 0 \\
\hline AdNAC26 & AdNTL3 & 551 & $523 \ldots . .545$ & 22.13992 & 0.00091 \\
\hline AdNAC36 & AdNTL4 & 592 & $569 \ldots . .591$ & 22.56383 & 0.01596 \\
\hline AiNAC12 & $\begin{array}{l}\text { AiNTL5, } \\
\text { AiNTL6 }\end{array}$ & 678 & $\begin{array}{l}582 \ldots \ldots 604 \\
656 \ldots .6675\end{array}$ & 41.84687 & 0 \\
\hline AiNAC16 & AiNTL7 & 216 & $5 \ldots \ldots 27$ & 20.81172 & 20.80519 \\
\hline AiNAC17 & AiNTL8 & 559 & $531 \ldots . .553$ & 22.14053 & 0.00084 \\
\hline AiNAC29 & $\begin{array}{l}\text { AiNTL9, } \\
\text { AiNTL10 }\end{array}$ & 583 & $\begin{array}{l}531 \ldots . .553 \\
558 \ldots . .580\end{array}$ & 40.01905 & 0 \\
\hline AiNAC37 & AiNTL11 & 698 & $669 \ldots . .691$ & 22.56604 & 0.01787 \\
\hline
\end{tabular}




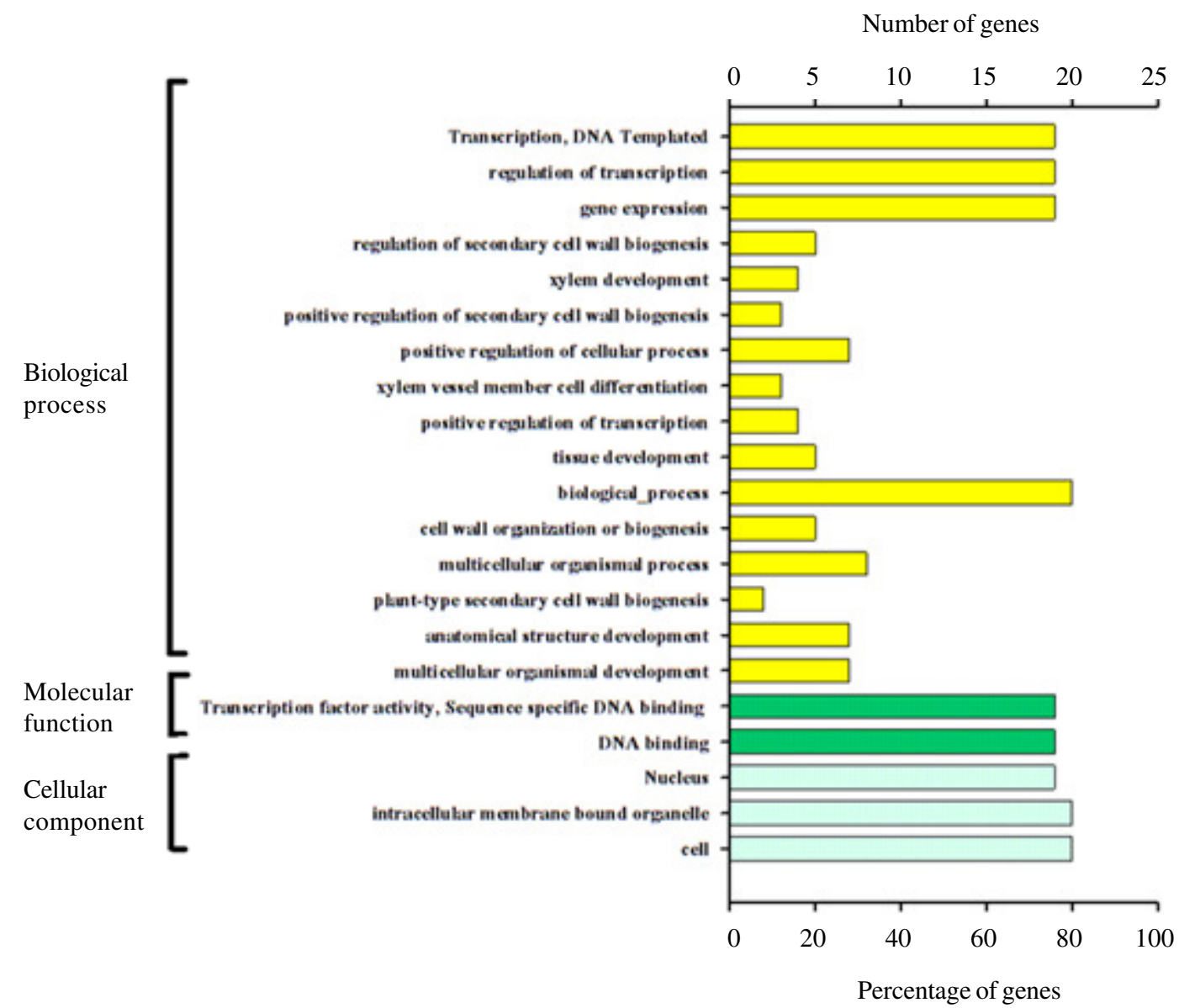

Figure 9. Gene Ontology annotation of NAC proteins in groundnut.

in biotic and abiotic stress responses. Considering the varied functions of membrane bound NAC genes in crops, the identification of four membrane bound NAC genes would be useful in understanding their specific functions in groundnut.

More than 14 cis elements were identified; among them most commonly found elements are depicted in Figure 11. Identification of MYB binding site and $\mathrm{W}$ - box in the promoter region, emphasize the role of MYB and WRKY TFs in regulating NAC TFs. Moreover, the presence of LTR, ABRE and HSE further envisages the function of NAC TFs in abiotic stress responses.
Expression profiles of $A d N A C$ and $A i N A C$ genes. To investigate the response of NAC TFs to drought and high temperature, the expression profiles of NAC genes in different tissues were analysed and the results are expressed as fold change with respect to the control. During drought stress in shoot, AdNAC32, AdNAC36, AdNAC38, AiNAC24, AiNAC32, AiNAC35,AiNAC37,AdNAC5, AdNAC6, AdNAC12, AdNAC15, AdNAC21, $A d N A C 22, A d N A C 24, A d N A C 29$ and AdNAC35 showed induced expression ranging between 1 to 4 fold (Figs. 12 and 13).

High temperature stress in shoot upregulated 48 genes, among them $A d N A C 7,22$, 


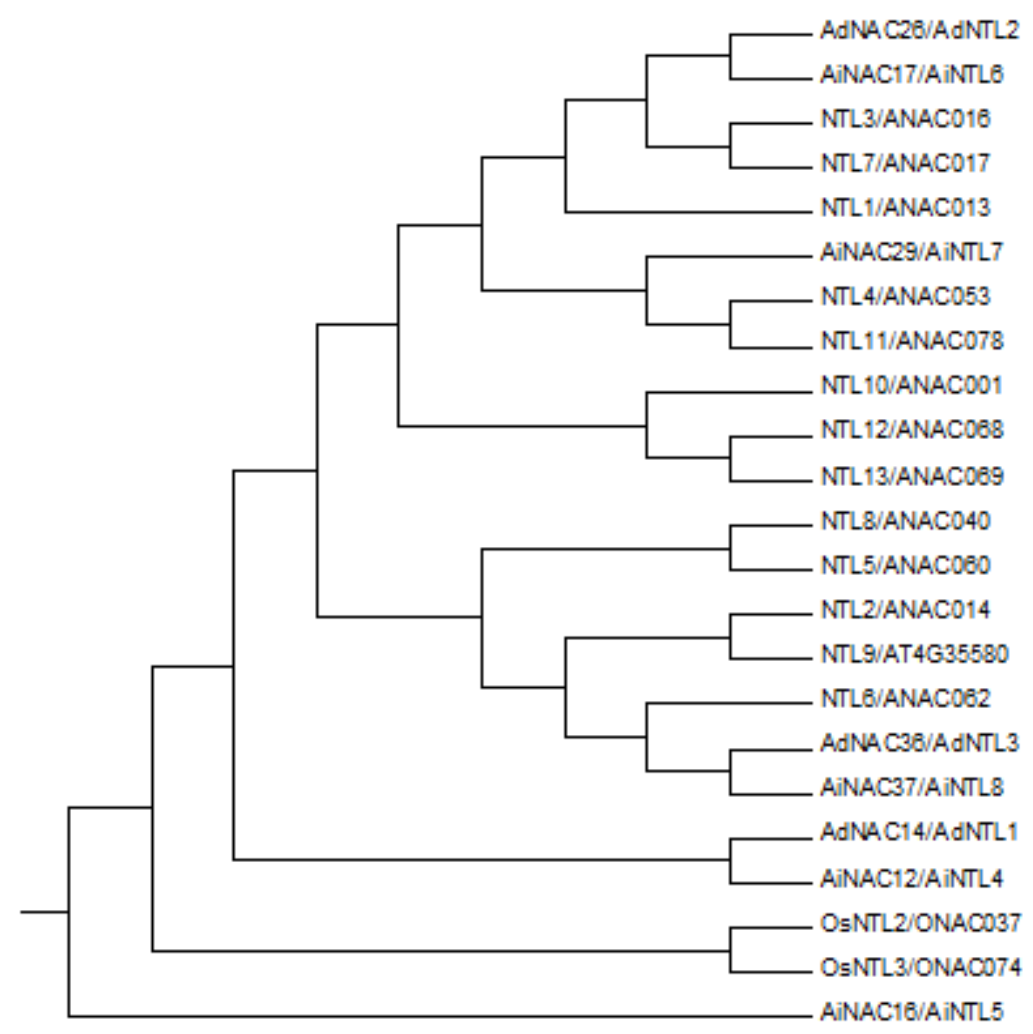

Figure 10. Phylogenetic tree of membrane-bound NACs from groundnut (AdNTLs\&AiNTLs/ AdNACs \& AiNACs), Arabidopsis (NTLs/ANACs) and rice (OsNTLs/ONACs). The unrooted phylogenetic tree was constructed using the full protein sequences.

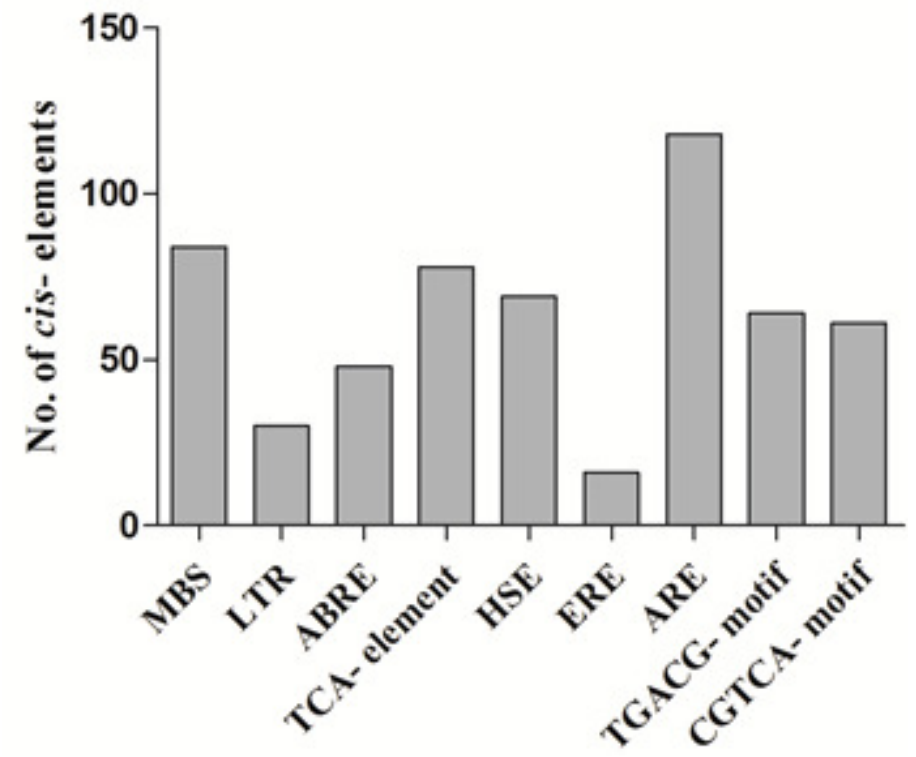

Figure 11. Different types of cis-elements identified in the promoter region of NAC TFs genes. 
AiNAC Drought

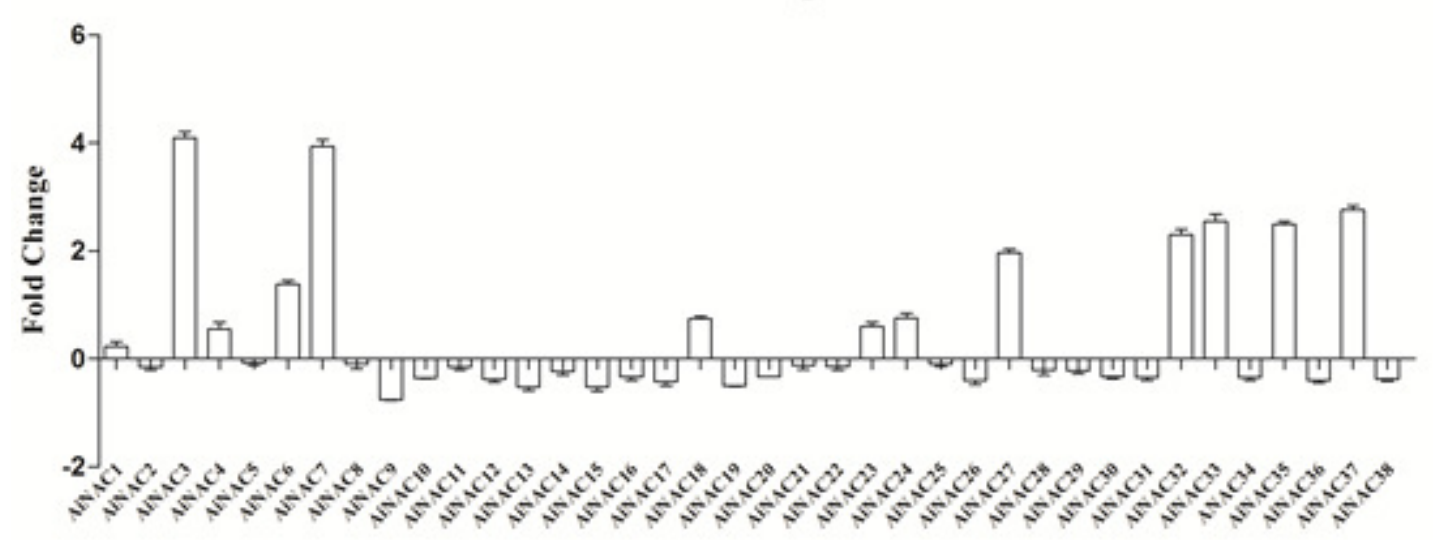

Figure 12. Expression profile of $A d N A C$ genes in shoot sample underdrought stress by RT-qPCR.

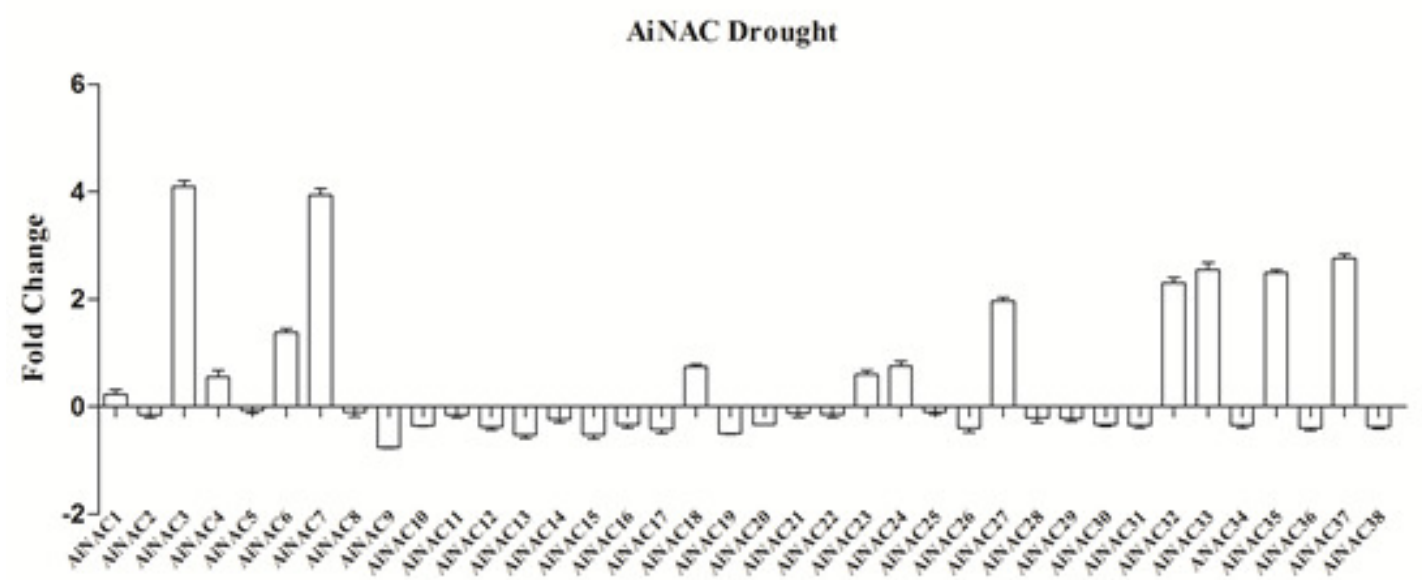

Figure 13. Expression profile of AiNAC genes in shoot sample underdrought stress by RT-qPCR.

32, AiNAC5, 6, 21 showed induced expression by $4.1,2.1,3.01,2.11,2.52,2.59$ fold, respectively; while the rest of the genes showed fold change between 1 and 3.However, the remaining 28 of 76 genes were down-regulated with the fold changes ranging between 0.5 and 1 (Figs. 14 and 15).

$A d N A C 21$ and AiNAC3 showed upregulation under both drought and high temperature stress; while AdNAC5, 7, 10, 18,20, 36 and AiNAC1, 6, 28, 30, 36, 38 exclusively showed their presence in shoot $A i N A C 33$ was found to be root specific in high temperature stressed seedlings only. In addition, AdNAC11,21,36, AiNAC8,23, 24,35, 37 were found in shoot; and AiNAC22,28, 29 were found to be root specific during drought (Figs. 16 and 17).

Our results reveal the involvement of AiNAC36 as a positive regulator under high temperature and drought conditions localised in shoot tissue. The differential expression of NAC genes obtained from qRT-PCR is represented as a heat map and clustered. The heat map generated for in-silico expression pattern, the differential transcript abundance 


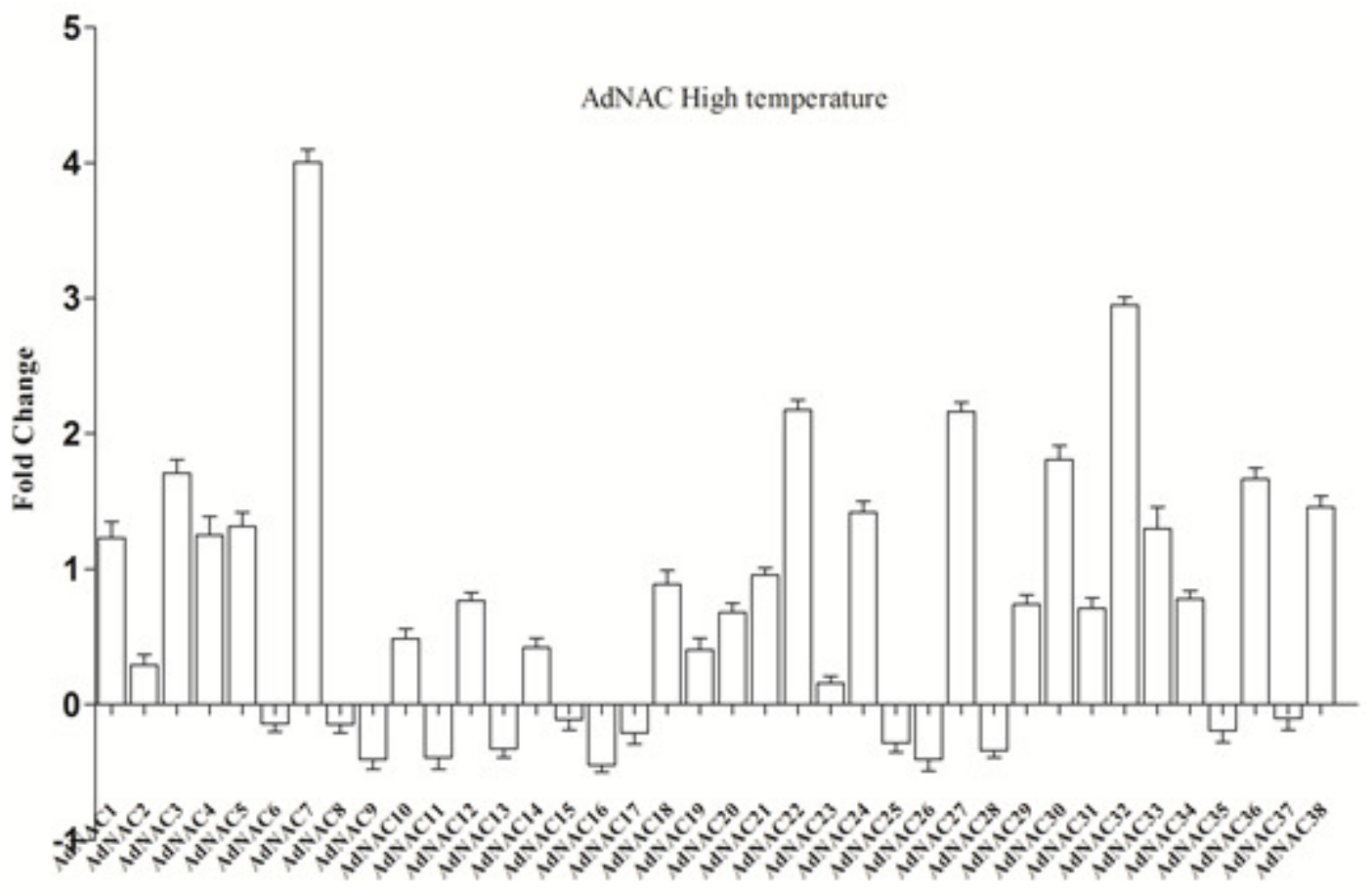

Figure 14. Expression profile of $A d N A C$ genes in shoot sample underhigh temperature stress by RT-qPCR.

of the NAC genes in the shoot and root tissue is consistent with the results obtained from qRT- PCR.

Several reports demonstrated that NAC genes are involved in regulating plant development atdifferent growth stages (He et al., 2005; Chuanzao et al., 2007; Nian et al., 2013). In Arabidopsis, ANAC002/ ATAF1 was induced by long-term treatment with ABA and/ or during age-dependent senescence. Overexpression of drought, salinity and abscisic acid induced ANAC019, ANAC055 and ANAC072 in Arabidopsis resulted in increased tolerance to drought (Tran et al., 2004). AdNAC21 belonging to group VI was found to be induced by both drought and high temperature stress treatments.

A membrane bound NAC protein encoded by ANAC069 was shown to regulate seed germination by integrating auxin and salt signals in Arabidopsis (Park et al., 2011). In Glycine max, GmNAC2, GmNAC3 and GmNAC4 were strongly induced by osmotic stress. GmNAC3 and GmNAC4 were also induced by Abscisic acid (ABA), Jasmonic acid (JA) and salinity, but differed in their response to cold conditions (Guilherme et al., 2009). In addition, GmNAC2 over-expressing tobacco lines were developed and found to be hypersensitive to drought, high salinity and cold stress, indicating that GmNAC2 functions as a negative regulator during abiotic stress (Hangxia et al., 2013). Over-expression of TaNAC2L in transgenic Arabidopsis upregulated the expression of heat related genes, suggesting that it plays a role in improving heat tolerance by regulating the expression of stress responsive genes (Guo et al., 2015).

In rice, SNAC3 was ubiquitously expressed and its transcript level was induced by drought, high temperature, salinity stress, and abscisic acid (ABA) treatment. Over-expression of SNAC3 in rice resulted in enhanced tolerance to high temperature, drought, and oxidative stress caused by methyl viologen (MV); whereas suppression of SNAC3 by RNAi 


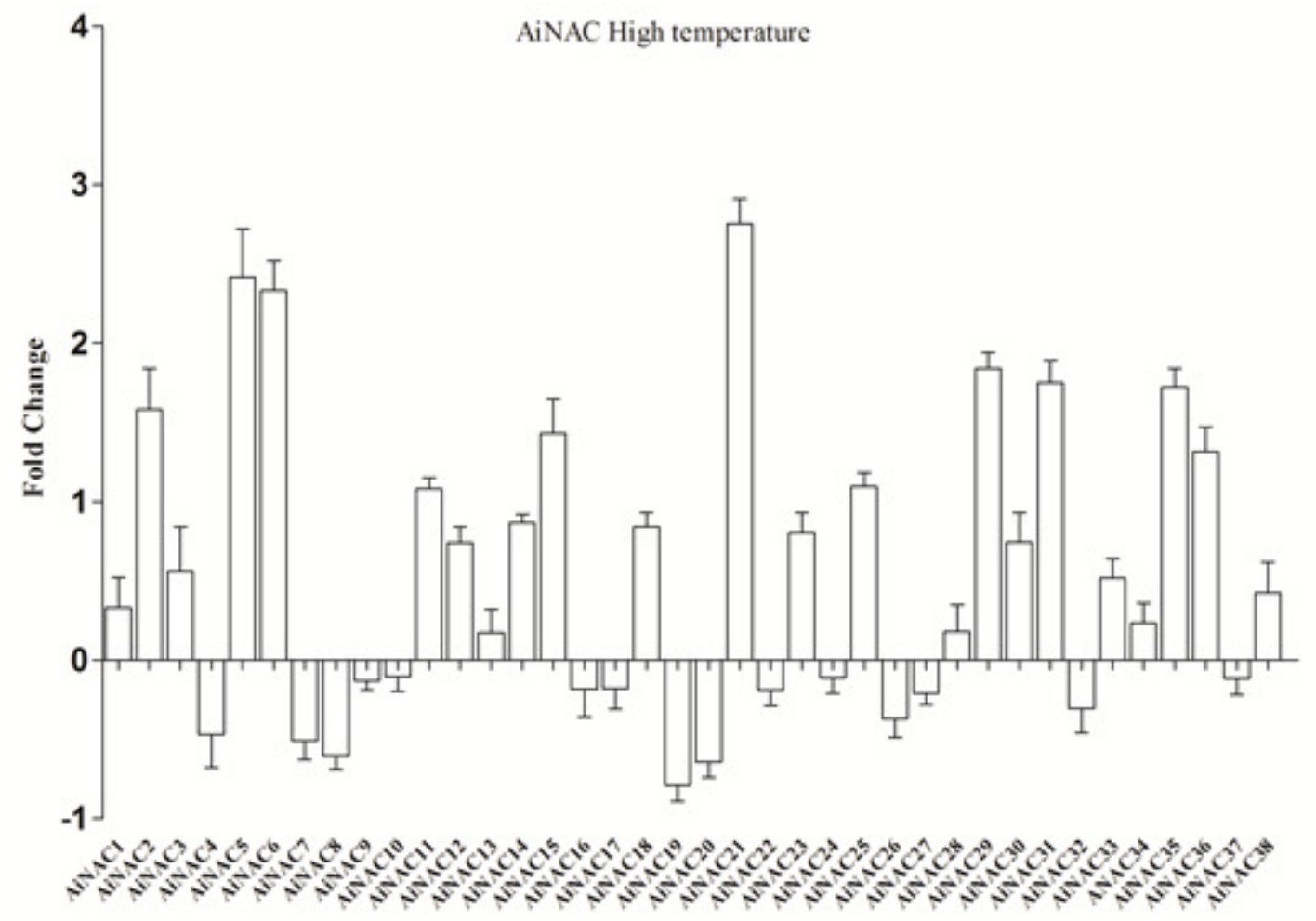

Figure 15. Heat map representation of differential expression of $A d N A C$ and $A i N A C$ genes in response to drought stress. $\mathrm{C}=$ Cotyledon, $\mathrm{L}=$ Leaf, $\mathrm{S}-\mathrm{Stem}$ and $\mathrm{R}=$ Root. The heat-map was generated based on the fold change values in the treated sample when compared to control sample. The color scale for fold change values is shown at the top.

resulted in increased sensitivity to these stresses (Yujie et al., 2015). In our study, $A d N A C 21$ and AiNAC3 were found to be upregulated under both drought and high temperature stress, suggesting that these genes may be positive regulators of abiotic stress responses in groundnut. The variability in gene expression patterns implies that NACs may regulate a complex web of pathways, to perform different physiological functions for acclimatising towards multiple challenges. Furthermore, the NAC genes were differentially expressed in a tissue specific manner under drought and high temperature stress conditions. This understanding will prove useful in improving drought and high temperature tolerance since these genes would be involved in abiotic tolerance in groundnut.

\section{CONCLUSION}

Genome wide expression patterns of 76 NAC transcription factors from groundnut have been unveiled. Furthermore, the NAC genes were differentially expressed in tissue specific manner under high temperature and drought. $A d N A C 21$ and AiNAC3 are significantly expressed under both the stress conditions. Thus, the analysis provides preliminary indications of putative functions of groundnut NAC transcription factors, which would help in channelising directional efforts for their functional characterisation. 


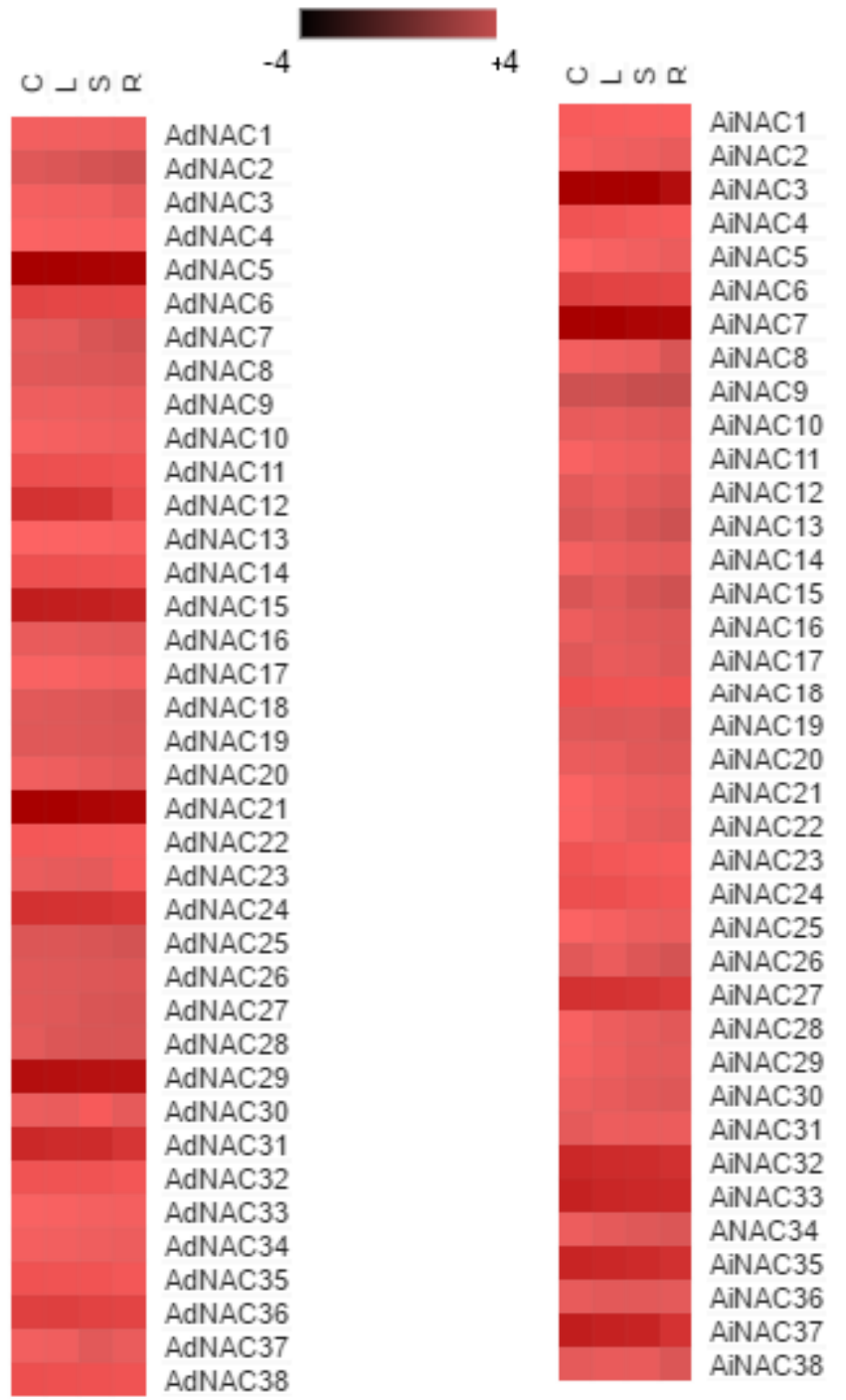

Figure 16. Heat map representation of differential expression of $A d N A C$ and $A i N A C$ genes in response to drought stress. $\mathrm{C}=$ Cotyledon, $\mathrm{L}=$ Leaf, $\mathrm{S}-\mathrm{Stem}$ and $\mathrm{R}=$ Root. The heat-map was generated based on the fold change values in the treated sample when compared to control sample. The color scale for fold change values is shown at the top. 


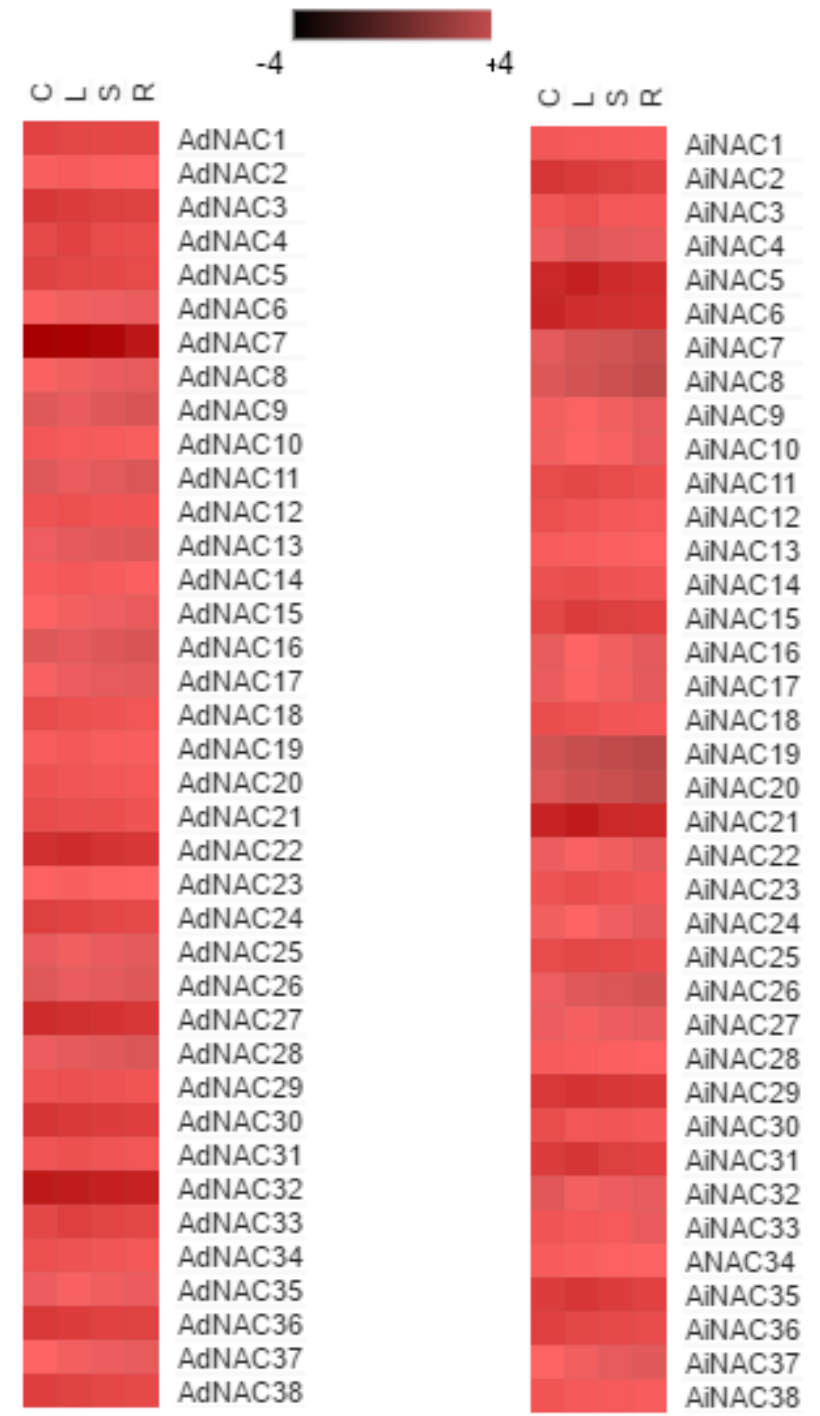

Figure 17. Heat map representation of differential expression of $A d N A C$ and AiNAC genes in response to high temperature stress. $\mathrm{C}=$ Cotyledon, $\mathrm{L}=\mathrm{Leaf}, \mathrm{S}=$ Stem and $\mathrm{R}=$ Root. The heat-map was generated based on the fold-change values in the treated sample when compared to control sample. The color scale for fold-change values is shown at the top. 


\section{ACKNOWLEDGEMENT}

This work was supported by funds from the Science and Engineering Research Board (Ref No: SB/EMEQ- 141/2013).

\section{REFERENCES}

Aslam, M., Grover, A., Sinha, V.B., Fakher, B., Pande, V. and Yadav, P.V. 2012. Isolation and characterization of cold responsive NAC gene from Lepidium latifolium. Molecular Biology Reporter 39:1-10.

Bailey, T.L., Boden, M. and Buske, F.A. 2009. MEME SUITE: Tools for motif discovery and searching. Nucleic Acids Research 37:202-208.

Bertiol, D.J., Seijo, G., Freitas, F.O., Valls, J.F.M., Leal-Bertioli, S.C.M. and Moretzsohn, M.C. 2011. An overview of peanut and its wild relatives. Plant Genetic Resource 9:134-149.

Chen, Q., Wang, Q., Xiong, L. and Lou, Z. 2011. A structural view of the conserved domain of rice stress-responsive NAC1. Protein Cell 2:55-63.

Chuanzao, M., Wona, D., Yunrong, W., Jie, Y., Xiaowei, H., Huixai, S. and Ping, W. 2007. Over-expression of a NAC-domain protein promotes shoot branching in rice. New Phytologist 2:288-298.

Guilherme, L., Pinheiro, Carolina, S. and Marques, 2009. Complete inventory of soybean NAC transcription factors: Sequence conservation and expression analysis uncover their distinct roles in stress response. Gene 444 (1-2):10-23.

Guo, W., Zhang, J. and Zhang, N. 2015. The wheat NAC transcription factor TaNAC2L is regulated at the transcriptional and posttranslational levels and promotes heat stress tolerance in transgenic arabidopsis. PLoSONE 10(8):e0135667.

Guo, Y. and Gan, S. 2006. AtNAP, a NAC family transcription factor, has an important role in leaf senescence. Plant Journal 46:601-612.

Ha, C.V., Nasr Esfahani, M., Watanabe, Y., Tran, U.T. and Sulieman, S. 2014. Genome-wide identification and expression analysis of the CaNAC family members in chickpea during development, dehydration and ABA treatments. PLoSOne 9(12): e114107.

Hall, T.A. 1999. BioEdit: A user-friendly biological sequence alignment editor and analysis program for Windows 95/98/NT. Nucleic Acids Symposium Series 41:95-98. Hangxia, J., Fang, H., Hao, C., Haina, S. and Deyue, Y. 2013. Overexpression of the GmNAC2 Gene, a NAC transcription factor, reduces abiotic stress tolerance in tobacco. Plant Molecular Biology Reporter $31: 435-442$

He, X.J., Mu, R.L., Cao, W.H., Zhang, Z.G., Zhang, J.S. and Chen, S.Y. 2005. AtNAC2, a transcription factor downstream of ethylene and auxin signaling pathways, is involved in salt stress response and lateral root development. Plant Journal 44:903-916.

Hobert, O. 2008 Gene regulation by transcription factors and microRNAs. Science 319:1785-1786.

Jeong, J.S., Kim, Y.S., Baek, K.H., Jung, H., Ha, S.H. and Do Choi, Y. 2010. Rootspecific expression of OsNAC10 improves drought tolerance and grain yield in rice under field drought conditions. Plant Physiology 153:185-197.

Kim, S.G., Lee, S., Seo, P.J., Kim, S.K., Kim, J.K. and Park, C.M. 2010. Genome-scale screening and molecular characterization of membrane-bound transcription factors in Arabidopsis and rice. Genomics 95(1):5665.

Kim, S.Y., Kim, S.G., Kim, Y.S., Seo, P.J. and Bae, M. 2007. Exploring membraneassociated NAC transcription factors in Arabidopsis: implications for membrane biology in genome regulation. Nucleic Acid Research 35:203-213. 
Kochert, G., Stalker, H., Gimenes, M., Galgaro, M., Lopes, C. and Moore, K. 1996. RFLP and cytogenetic evidence on the origin and evolution of allo-tetraploid domesticated peanut, Arachishypogaea (Leguminosae). American Journal of Botany 83:1282-1291.

Kou, X.H., Wang, S., Wu, M.S., Guo, R.Z. and Xue, Z.H. 2014. Molecular characterization and expression analysis of NAC family transcription factors in tomato. Plant Molecular Biology Reporter 32:501516.

Le, D.T., Nishiyama, R., Watanabe, Y., Mochida, K. and Yamaguchi-Shinozaki, K. 2011. Genome-wide survey and expression analysis of the plant-specific NAC transcription factor family in soybean during development and dehydration stress. DNA Research 18:263-276.

Liu, T., Song, X., Duan, W., Huang, Z. and Liu, G. 2014. Genome-wide analysis and expression patterns of NAC transcription factor family under different developmental stages and abiotic stresses in Chinese cabbage. Plant Molecular Biology Reporter 32:1041-1056.

Mohammed, N., Akhter, M., Sharoni and Shoshi, K. 2013. Roles of NAC transcription factors in the regulation of biotic and abiotic stress responses in plants. Frontiers in Microbiology 3(4): 248.

Nagy, E.D., Guo, Y., Tang, S., Bowers, J.E., Okashah, R.A. and Taylor, C.A. 2012. A high-density genetic map of Arachis duranensis, a diploid ancestor of cultivated peanut. BMC Genomics 13:469.

Nian, W., Yu, Z., Haiping, X., Linchuan, F. and Shaohua, L. 2013. Comprehensive analysis of NAC domain transcription factor gene family in Vitis vinifera. Plant Cell Reports 32:61-75.

Oh, S.K., Lee, S., Yu, S.H. and Choi, D. 2005. Expression of a novel NAC domaincontaining transcription factor (CaNAC1) is preferentially associated with incompatible interactions between chili pepper and pathogens. Planta 222:876887.

Ooka, H., Satoh, K., Doi, K., Nagata, T. and Otomo, Y. 2003. Comprehensive analysis of NAC family genes in Oryza sativa and Arabidopsis thaliana. DNA Research 10:239-247.

Park, J., Kim, Y.S., Kim, S.G., Jung, J.H., Woo, J.C. and Park, C.M. 2011. Integration of Auxin and Salt Signals by the NAC Transcription Factor NTM2 during Seed Germination in Arabidopsis. Plant Physiology 156 (2):537-549.

Puranik, S., Sahu, P.P., Srivastava, P.S. and Prasad, M. 2012. NAC proteins: regulation and role in stress tolerance. Trends Plant Science 17:369-381.

Puranik, S., Sahu, P.P., Mandal, S.N.,Venkata, S.B. and Parida, S.K. 2013. Comprehensive genome-wide survey, genomic constitution and expression profiling of the NAC transcription factor family in foxtail millet (Setaria italica L.) PLoS One 8:e64594.

Ren, T., Wang, J., Zhao, M., Gong, X., Wang, S., Wang, G. and Zhou, C. 2017. Involvement of NAC transcription factor SiNAC1 in a positive feedback loop via ABA biosynthesis and leaf senescence in foxtail millet. Planta 247(1):53-68.

Sablowski, R.W.M. and Meyerowitz, E.M. 1998. A homolog of no apical meristem is an immediate target of the floral homeotic genes APETALA3/PISTILLATA. Cell 92:93-103.

Seijo, G., Lavia, G.I., Fernandez, A., Krapovickas, A., Ducasse, D.A. and Bertioli, D.J. 2007. Genomic relationships between the cultivated peanut (Arachis hypogaea, Leguminosae) and its close relatives revealed by double GISH. American Journal of Botany 94:19631971.

Seo, P.J. and Park, C.M. 2010. A membranebound NAC transcription factor as an integrator of biotic and abiotic stress 
signals. Plant Signalling \& Behaviour 5(5):481-483.

Shiriga, K., Sharma, R., Kumar, K., Yadav, S.K. and Hossaina, F. 2014. Genome-wide identification and expression pattern of drought-responsive members of the NAC family in maize. Meta Gene 2:407-417.

Singh, A.K., Sharma, V., Pal, A.K., Acharya, V. and Ahuja, P.S. 2013. Genome-wide organization and expression profiling of the NAC transcription factor family in potato (Solanum tuberosum L.). DNA Research 20:403-423.

Tamura, K., Stecher, G., Peterson, D., Filipski, A. and Kumar, S. 2013. MEGA6: Molecular Evolutionary Genetics Analysis Version 6.0. Molecular Biology and Evolution 30 (12):2725-2729.

Tran, L.S., Nakashima, K., Sakuma, Y., Simpson, S.D., Fujita, Y. and Maruyama, K. 2004. Isolation and functional analysis of Arabidopsis stress inducible NAC transcription factors that bind to a drought responsive cis-element in the early responsive to dehydration stress 1 promoter. Plant Cell 16:2481-2498.
Wang, X., Basnayake, B.M., Zhang, H., Li, G., Li, W. and Virk, N. 2009. The Arabidopsis ATAF1, a NAC transcription factor, is a negative regulator of defense responses against necrotrophic fungal and bacterial pathogens. Molecular Plant Microbe Interactions 22:1227-1238.

Yujie, F., Kaifeng, L., Hao, D., Yan, X., Huazhi, S., Xianghua, L. and Lizhong, X. 2015. A stress-responsive NAC transcription factor SNAC3 confers heat and drought tolerance through modulation of reactive oxygen species in rice. Journal of Experimental Botany 66 (21):68036817.

Zheng, X., Chen, B., Lu, G. and Han, B. 2009. Overexpression of a NAC transcription factor enhances rice drought and salt tolerance. Biochemical and Biophysical Research Communications 379:985-989.

Zhenying, W., Xueqin, X., Wangdan, X., Pingzhi, W., Yaping, C., Meiru, L., Guojiang, W. and Huawu, J. 2015. Genome-wide analysis of the NAC gene family in physic nut (Jatropha curcas L.). PLoSOne 10(6):e0131890. 